\title{
Über den Einfluß elektrischer Felder auf die Lebensdauer des metastabilen Niveaus des Wasserstoffatoms ${ }^{1}$
}

\author{
Von Gerhart Lüders \\ Aus dem Institut für Theoretische Physik der Universität Hamburg \\ (Z. Naturforschg. 5 a, 608-611 [1950]; eingegangen am 10. Oktober 1950)
}

\begin{abstract}
Es wird eine Neuberechnung des Einflusses elektrischer Felder auf die Lebensdauer des metastabilen Niveaus des Wasserstoffatoms unter Berücksichtigung der L a m b-shift vorgelegt. Durch die Lamb-shift wird die mittlere Lebensdauer zwischen 0,03 und $10 \mathrm{Volt}^{\mathrm{cm}^{-1}} \mathrm{um}^{\mathrm{mehr}}$ als zwei Zehnerpotenzen verlängert.
\end{abstract}

$\mathrm{D}$ ie Metastabilität des $2 \mathrm{~S}_{1 / 2}$-Niveaus des Wasserstoffatoms beruht bekanntlich darauf, daß infolge der $l$-Auswahlregel ein mit elektrischer Dipolstrahlung verbundener Übergang in den Grundzustand verboten ist. Nach den Untersuchungen von B re it und T e ll e $\mathrm{r}^{2}$ ist ein Übergang in den Grundzustand am wahrscheinlichsten, wenn er mit der gleichzeitigen Emission von 2 Lichtquanten verbunden ist. Die mittlere Lebensdauer des $2 \mathrm{~S}_{12}$-Niveaus ergibt sich dabei zu etwa $1 /$ s sec.

Ein äußeres elektrisches Feld vermischt den 2 SZustand mit 2 P-Zuständen und kann daher bei hinreichender Stärke die Lebensdauer, d.h. die Metastabilität, wesentlich vermindern. Bei Gültigkeit der S o m merfeldschen Feinstrukturformel, nach der Niveaus mit gleicher Hauptquantenzahl $n$ und gleicher Drehimpulsquantenzahl $j$ genau zusammenfallen, würde bereits ein schwaches äußeres Feld die beiden Zustände $j=1 / 2, l=0$ und $j=1 / 2, l=1$ völlig vermischen und die Lebensdauer beträchtlich herabsetzen. Allerdings sind Abweichungen von dieser Überlegung zu erwarten, solange die durch das elektrische Feld hervorgerufene Aufspaltung von der Größenordnung der natürlichen Niveaubreite ist (vgl. ${ }^{3}$ ).

Nun ist vor einigen Jahren experimentell nachgewiesen ${ }^{4}$ und theoretisch verständlich gemacht ${ }^{5}$ worden, daß die Feinstrukturformel nicht in Strenge zutrifft. Diese Abweichung, die Lamb-shift, hat ihre Ursache in der Wechselwirkung des Elektrons mit dem Strahlungsfeld und führt u. a. dazu, daß der $2 S_{1}$-Term höher liegt als der $2 \mathrm{P}_{12}$-Term. B e the

1 Teilweise enthalten in der unveröffentlichten Hamburger Dissertation (1950) des Verf.

2 G. B reit u. E. T e lle r, Astrophysic. J. 91, 215 [1940].

3 H. B ethe in Hdb. Physik, 2. Aufl., Bd. 24/1, 273, Berlin 19:33. und Mitarbb. ${ }^{6}$ errechnen für diese Verschiebung einen Wert von $1051 \mathrm{MHz}$.

Durch die Lamb-shift wird die Empfindlichkeit des metastabilen Nivcaus in entscheidender Weise geändert. Hinreichend schwache Felder führen, da der $2 \mathrm{~S}_{1 \text { 2 }}$-Term und der $2 \mathrm{P}_{12 \text { - }}$-Term nicht mehr zusammenfallen, zu einer nur geringfügigen P-Beimischung zu dem S-Zustand und daher zu einer nur geringfügigen Verminderung der Lebensdauer.

Ziel dieser Arbeit ist es, eine Neuberechnung des Einflusses elektrischer Felder auf die Lebensdauer des metastabilen Niveaus des Wasserstoffatoms unter Berücksichtigung der Lamb-shift vorzulegen. Eine Berücksichtigung der natürlichen Linienbreite, die bei früheren Untersuchungen eine große Rolle spielte, erweist sich dabei als unnötig, da das S-Niveau und das P-Niveau bereits ohne äußeres Feld einen Abstand haben, der hinreichend groß gegen die natüriche Niveaubreite ist. Der durch diese Vernachlässigung begangene Fehler beträgt auch bei kleinsten Feldstärken weniger als 1 Prozent.

Um ein genaueres Urteil über die Rolle der Lambshift zu ermöglichen, wird dem Ergebnis dasjenige gegenübergestellt, das sich ohne Lamb-shift ergeben würde.

Durchführung der Rechnung

Ein angeregtes Niveau eines Atoms klingt unter Emission von elektrischer Dipolstrahlung mit einer Zeitkonstanten $\gamma$, der reziproken mittleren Lebensdauer, ab, die gegeben ist durch

4 W. E. Lamb jr. u. R. Retherford, Physic. Rev. 72, $241[1947]$.

5 H. A. B ethe, Physic. Rev. 72, 339 [1947]; T. A. We lt o n, Physic. Rev. 74, 1157 [1948].

${ }^{6}$ H. A. Bethe, L. M. Brown u. J. R. Stehn, Physic. Rev. 77, 370 [1950]. 


$$
\gamma=\sum_{\mathrm{b}} \gamma_{\mathrm{ab}}
$$

mit

$$
\gamma_{\mathrm{ab}}=\begin{array}{cc}
4 & e^{2} \\
3 & \hbar
\end{array}\left(\frac{\omega_{\mathrm{ab}}}{c}\right)^{3}\left(\mathrm{r}_{\mathrm{ab}}^{*} \mathrm{r}_{\mathrm{ab}}\right)
$$

(vgl. ${ }^{3}$ ). In (1) ist über alle vom Anfangszustand a aus erreichbaren energetisch tieferen Endzustände b zu summieren. In (2) stellen die $\omega_{\mathrm{a} b}$ die Kreisfrequenzen der Ưbergänge und die $\mathfrak{r}_{a b}$ die zugehörigen Matrixelemente des Ortsvektors $\mathfrak{r}$ dar. Von dem hier interessierenden $2 \mathrm{~S}_{1 / 2}$-Niveau aus sind, da der Grundzustand zweifach entartet ist, zwei Übergangsmöglichkeiten vorhanden. Ohne äußeres Feld sind diese allerdings als elektrische Dipolübergänge verboten: die Matrixelemente $r_{a b}$ verschwinden.

Durch ein äußeres homogenes elektrisches Feld wird die Übergangsfrequenz in einer hier vernachlässigbaren Weise geändert, gleichzeitig aber erhalten die Matrixelemente infolge Änderung der Eigenfunktionen einen von Null verschiedenen Wert. Die hierdurch bedingte Abhängigkeit der Größe $\gamma$ vom äußeren elektrischen Feld $F$ gilt es nunmehr für das metastabile Niveau zu errechnen. Hierfür ist mittels einer Störungsrechnung zunächst die Energieänderung (S t a r k - Effekt) und anschließend die Änderung der Matrixelemente $\mathfrak{r}$ für die Úbergänge von diesem $\mathrm{Zu}$ stand aus zu gewinnen.

Die Methode der Störungsrechnung wird an anderer Stelle dargestellt (vgl. ${ }^{7}$, insbesondere Ziffer 3). Genauer zu untersuchen sind danach nur die Terme mit $m_{j}=1 / 2$ (bzw. die damit zusammenfallenden mit $\left.m_{j}=-1 / 2\right)$. Die Säkulardeterminante $\left(16_{1}\right)$ in 7 ist jetzt so abzuändern, daß die Lamb-shift mitberücksichtigt wird. Hierfür braucht die Wechselwirkung mit dem Strahlungsfeld bei Anwesenheit eines äußeren elektrischen Feldes nicht erneut berechnet zu werden, da die der Lamb-shift entsprechende Zusatzenergie nach $^{5}$ gleich dem Erwartungswert von $\Delta\left(e^{2} / r\right)(\Delta=$ Laplace-Operator) multipliziert mit einem von $n$ abhängigen Faktor ist. Die Lamb-shift kann also dadurch berücksichtigt werden, daß zu den $n$-Teilmatrizen der verschiedenen Anteile des Energieoperators $\left(\right.$ vgl. ${ }^{7}$ ) die $n$-Teilmatrix von $\Delta \frac{1}{r}=4 \pi \delta(\mathfrak{r})$ mit einem geeigneten Zahlenfaktor addiert wird. Dieser Faktor ist so zu wählen, daß der S-Term im feldfreien Fall eine Verschiebung von $1051 \mathrm{MHz}$ erhält.

7 G. L ü d e r s, Der Stark-Effekt des Wasserstoffs bei kleinen Feldstärken, Ann. Physik (im Erscheinen).
Die durch ein elektrisches Feld der Stärke $F$ hervorgerufene Energieänderung $\delta E$, bezogen auf das Niveau mit $j=3 / 2$ als Nullpunkt, ergibt sich dann als Lösung der folgenden Säkulardeterminante

$$
\left|\begin{array}{ccc}
-\xi & 0 & A \sqrt{6} \\
0 & -16-\xi & -A \sqrt{3} \\
A \sqrt{6} & -A \sqrt{3} & -\frac{1}{16}-\xi
\end{array}\right|=0
$$

und der daraus folgenden algebraischen Gleichung

$\xi^{3}+\xi^{2}\left(\frac{1}{8}-\varepsilon\right)+\xi\left(\frac{1}{256}-\frac{\varepsilon}{16}-9 A^{2}\right)-{ }_{8}^{3} A^{2}=0$.

Dabei sind folgende dimensionslosen Größen benutzt worden

$$
A=\frac{e \mathbf{a} F}{a^{2} e^{2} / 2 \mathbf{a}}, \quad \xi=\frac{\delta E}{t^{2} e^{2} / 2 \mathbf{a}} .
$$

Die Größe $\varepsilon$ stellt die Lamb-shift, gemessen ebenfalls in Einheiten $\alpha^{2} e^{2} / 2 \mathbf{a}$, dar und hat bei Zugrundelegung einer Verschiebung von $1051 \mathrm{MHz}$ den Zahlenwert $6,002 \cdot 10^{-3} . \mathbf{a}=\hbar^{2} / m_{0} e^{2}$ bedeutet den Radius der innersten $\mathrm{B}$ oh r schen Bahn, $\alpha=e^{2} / \hbar c$ die $\mathrm{S}$ o m m e r f e ld sche Feinstrukturkonstante.

In der Säkulardeterminante (3) bezieht sich die erste Zeile und Spalte auf die Funktion mit $j=3 / 2, l=1$, die zweite Zeile und Spalte auf die Funktion mit $j=1 / 2, l=1$ und die dritte auf $j=1 / 2, l=0$. Die ungestörten Funktionen sollen in der genannten Reihenfolge, in Übereinstimmung mit ${ }^{7}$, mit $\psi_{2}, \psi_{3}, \psi_{4}$ bezeichnet werden.

Wenn man sich lediglich auf die Untersuchung der bei Anlegen eines Feldes aus den Niveaus mit $j=1 / 2$ hervorgehenden Terme beschränkt, so genügt es, die Energieänderung bei hinreichend kleinen Feldern aus der folgenden Determinante

$$
\left|\begin{array}{cc}
-\frac{1}{16}-\xi & -A \sqrt{3} \\
-A \sqrt{3} & -\frac{1}{16}-\xi
\end{array}\right|=0
$$

d. h. der Gleichung

$$
\left(\xi+\frac{1}{16}\right)^{2}-\varepsilon\left(\xi+\frac{1}{16}\right)-3 A^{2}=0
$$

$\mathrm{zu}$ berechnen. Löst man (7) für hinreichend kleine Felder durch die ersten Glieder einer Reihenentwicklung; so gilt schließlich für den aus dem metastabilen 
Niveau hervorgehenden Term

$$
\xi+\frac{1}{16}=\varepsilon+\frac{3}{\varepsilon} A^{2}+\ldots .
$$

Einen Überblick über den Verlauf der Größe $\xi$ (bzw. der Verschiebung in $\mathrm{cm}^{-1}$ ) als Funktion der Feldstärke $F$ gibt Abb. 1, in der zum Vergleich derjenige Verlauf gestrichelt eingetragen ist, der sich ohne Lamb-shift ergeben würde. Unterhalb einer Feldstärke von etwa $200 \mathrm{Volt} \mathrm{cm}^{-1}$ wurde der Verlauf der von $j=1 / 2$ ausgehenden Terme mittels (7) berechnet. Da bei $j=1 / 2$ der S-Term $(l=0)$ und der P-Term

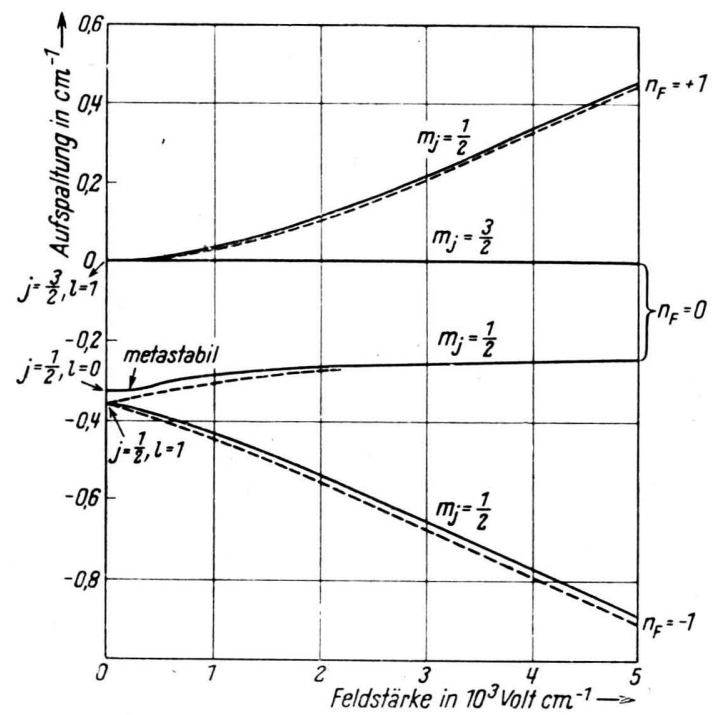

Abb. 1. Termverschiebung des Niveaus $n=2$ als Funktion der elektrischen Feldstärke ohne (---) und mit (- - ) Berücksichtigung der Lamb-shift.

$(l=1)$ ohne Feld nicht mehr genau zusammenfallen, erhält man zunächst statt des linearen einen quadratischen Anstieg [vgl. (8)]. Im Falle großer Feldstärken ergibt sich infolge der Lamb-shift eine Verschiebung der äußeren Terme $\left(n_{\mathrm{F}}= \pm 1\right)$ um die Hälfte $\left(0,017 \mathrm{~cm}^{-1}\right)$ der Verschiebung ohne Feld.

In Abb. 1 ist außerdem ein von $j=3 / 2$ nach $n_{\mathrm{F}}=0$ gehender, zu $m_{j}=3 / 2$ gehörender Term eingetragen, auf den die Lamb-shift keinen Einfluß hat.

Nachdem der Verlauf von $\xi$ (d. h. von der Energieverschiebung $\delta E$ ) für den vom metastabilen Niveau ausgehenden Term errechnet worden ist, läßt sich die Änderung der Größe $\gamma$ durch ein äußeres Feld gewinnen.

Die zum metastabilen Niveau gehörige Eigenfunktion $\psi_{4}$ wird nämlich bei Anlegen des Feldes $F$ ge- ändert in

$$
\varphi=a_{2} \psi_{2}+a_{3} \psi_{3}+a_{4} \psi_{4},
$$

wobei sich die feldstärkeabhängigen Entwicklungskoeffizienten $a_{k}$ bis auf einen gemeinsamen Normierungsfaktor $N$ aus den Unterdeterminanten zu einer (also z. B. der ersten) Zeile von (3) errechnen

$$
\begin{aligned}
& N a_{2}=\left|\begin{array}{cc}
-\frac{1}{16}-\xi-A \sqrt{3} \\
-A \sqrt{3} & \varepsilon-\frac{1}{16}-\xi
\end{array}\right|, \\
& N a_{3}=-\left|\begin{array}{cc}
0 & -A \sqrt{3} \\
A \sqrt{6} & \varepsilon-\frac{1}{16}-\xi
\end{array}\right|, \\
& N a_{4}=\left|\begin{array}{cc}
0 & -\frac{1}{16}-\xi \\
A \sqrt{6} & -A \sqrt{3}
\end{array}\right| .
\end{aligned}
$$

$N$ ist so zu bestimmen, daß gilt

$$
\left|a_{2}\right|^{2}+\left|a_{3}\right|^{2}+\left|a_{4}\right|^{2}=1 .
$$

In (10) ist für $\xi$ die entsprechende Lösung von (4) einzusetzen.

Im Rahmen der Näherung (7) kann, indem man die Unterdeterminanten der Determinante (6) entnimmt, gesetzt werden

$$
\begin{aligned}
a_{2}=0, \quad a_{3} & =\frac{\frac{1}{16}-\varepsilon+\xi}{\sqrt{\left(\frac{1}{16}-\varepsilon+\xi\right)^{2}+3 A^{2}}}, \\
a_{4} & =\frac{\sqrt{3} A}{\sqrt{\left(\frac{1}{16}-\varepsilon+\xi\right)^{2}+3 A^{2}}} .
\end{aligned}
$$

Die in (2) einzusetzenden, infolge des Einflusses des äußeren Feldes abgeänderten Matrixelemente $\mathfrak{r}_{a b}$ für die Übergänge zum Grundzustand errechnen sich nunmehr wegen (9) zu

$\int \varphi^{*} \mathrm{r}_{\mathrm{b}} d \tau=a_{2}{ }^{*} \int \psi_{2}{ }^{*} \mathrm{r} \%_{\mathrm{b}} d \tau+a_{3}{ }^{*} \int \psi_{3}{ }^{*} \mathrm{r} \%_{\mathrm{b}} d \tau$.

Dabei beziehen sich die Funktionen $\psi_{2}, \psi_{3}$, wie oben angegeben, auf den Anfangszustand $n=2$, während $\chi_{1}$ und $\chi_{2}$ die beiden Eigenfunktionen $\left(m_{j}=+1 / 2\right.$ bzw. $m_{j}=-1 / 2$ ) des durch ein Feld übrigens nicht beeinflußten Grundzustandes $n=1$ bedeuten. Das Matrixelement $\int \psi_{4}{ }^{*} \mathfrak{r}_{\gamma_{b}} d \tau$ tritt wegen der $l$-Auswahlregel in (13) nicht auf. Die übrigen Matrixelemente sind durch die nachfolgende Aufstellung gegeben (vgl. z. B. ${ }^{3}$ ). 


$$
\begin{aligned}
& \int \psi_{2}^{*} \mathbf{r} \psi_{1} d \tau=\frac{2^{8}}{3^{11 / 2}} \mathbf{a} z_{0}, \\
& \int \psi_{2}^{*} \mathfrak{r}_{2} d \tau=-\frac{2^{7}}{3^{11 / 2}} \mathbf{a}\left(\mathfrak{x}_{0}-i \eta_{0}\right), \\
& \int \psi_{3}{ }^{*} \mathfrak{r} \% 1 d \tau=-\frac{2^{15 / 2}}{3^{11 / 2}} \mathbf{a}_{z_{0}}, \\
& \int \psi_{3}{ }^{*} \mathfrak{r} \%_{2} d \tau=-\frac{2^{15 / 2}}{3^{11 / 2}} \mathbf{a}\left(\mathfrak{x}_{0}-i \mathfrak{y}_{0}\right) .
\end{aligned}
$$

$\mathfrak{x}_{0}, \mathfrak{y}_{0}, \mathfrak{z}_{0}$ bedeuten die Einheitsvektoren in der $x$-, $y$-, $z$-Richtung (Feld $\| \hat{z}_{0}$ ). Aus (13) und (14) folgt zunächst unter Benutzung der Normierungsbedingung (11)

$\left|\int \varphi^{*} \mathfrak{r} \%_{1} d \tau\right|^{2}+\left|\int \varphi^{*} \mathfrak{r} \%_{2} d \tau\right|^{2}=\frac{2^{15}}{3^{10}} \mathbf{a}^{2}\left(1-\left|a_{4}\right|^{2}\right)$.

Drückt man jetzt in (2) die Úbergangsfrequenz mittels der R y d b e r g - Formel durch die atomaren Konstanten aus und benutzt die Abkürzung

$$
\Gamma=\left(\frac{2}{3}\right)^{8} c^{4} \frac{c}{\mathrm{a}}=6,27 \cdot 10^{8} \mathrm{sec}^{-1},
$$

so gilt schließlich für die durch das Feld geänderte reziproke mittlere Lebensdauer für Dipolübergänge von dem metastabilen Niveau des Wasserstoffs aus die nachfolgende Beziehung

$$
\gamma=\Gamma\left(1-\left|a_{4}\right|^{2}\right) \text {. }
$$

Ohne Feld $(A=0)$ hat $a_{4}$ den Wert 1 und nimmt für starke Felder $(A \gg 1)$ asymptotisch den Wert Null an, da in (10) $N a_{2}$ und $N a_{3}$ bei starken Feldern proportional $A^{2}, N a_{4}$ aber proportional $A$ ist. $\gamma$ ändert sich also bei Anlegung eines wachsenden Feldes von Null bis $T$.

In der Abb. 2 ist dieser Verlauf in doppelt-logarithmischem Maßstab aufgetragen, wobei zu dem gemäß (17) berechneten $\gamma$-Wert der von B reit und Te $\mathrm{l}$ le $\mathrm{r}^{2}$ errechnete $\gamma$-Wert von $7 \mathrm{sec}^{-1}$ für Übergänge unter Emission zweier Quanten addiert wurde. Die hierdurch hervorgerufene Krümmung der Kurve macht sich in der Abbildung erst unterhalb 0,2 Volt $\mathrm{cm}^{-1}$ bemerkbar. Gl. (17) wurde unterhalb 50 Volt $\mathrm{cm}^{-1}$ mittels der aus (12) und (8) folgenden Näherung

$$
\gamma=3\left(\frac{A}{\varepsilon}\right)^{2} \Gamma=4,45 \cdot 10^{-6}\left(\frac{F}{\text { Volt cm }}\right) \cdot r
$$

ausgewertet. Unterhalb 300 Volt $\mathrm{cm}^{-1}$ wurde mit der Näherung (12) und (7) und oberhalb 300 Volt $\mathrm{cm}^{-1}$ mit der vollständigen Beziehung (10) gerechnet.
Um die Rolle zu verdeutlichen, die die Lamb-shift bei der Verlängerung der mittleren Lebensdauer spielt, ist in der gleichen Abbildung derjenige Verlauf von $\gamma$ als Funktion der Feldstärke aufgetragen, der sich bei Fehlen der Lamb-shift ergeben würde. Es genügt hierfür nicht, die ganze Rechnung in der gleichen Weise zu wiederholen, und nur $\varepsilon$ gleich Null zu setzen, insbesondere also (17) mit entsprechend abgeändertem $a_{4}$ beizubehalten. Wie bereits am Anfang der Arbeit betont wurde, sind die Abweichungen, die von der natürlichen Breite des $2 \mathrm{P}_{1 / 2}$-Niveaus herrühren, zu berücksichtigen.

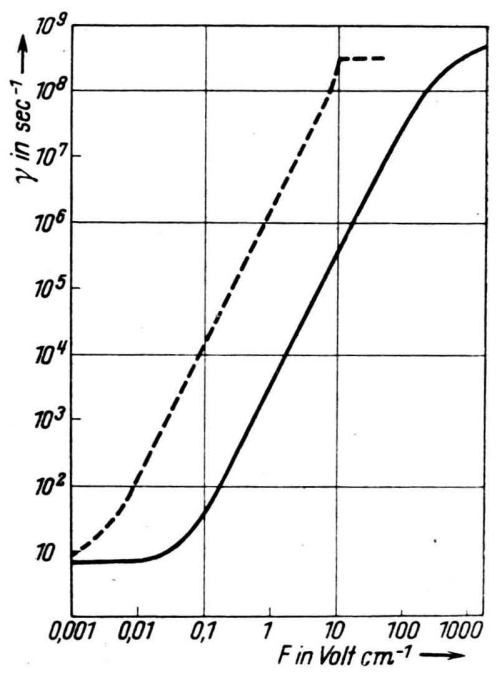

Abb. 2. Die reziproke mittlere Lebensdauer des metastabilen Niveaus als Funktion der Feldstärke (----) ohne und (-) mit Berücksichtigung der Lamb-shift.

Es ergibt sich dann (vgl. $\left.{ }^{3}\right)$ die folgende, mindestens bis 20 Volt $\mathrm{cm}^{-1}$ mit guter Näherung gültige Beziehung

$$
\gamma=\frac{1}{2}\left(1-\Re \mathrm{e} / 1-48\left(\frac{e \overline{\mathbf{a} F}}{\hbar \Gamma}\right)^{2}\right) \Gamma
$$

$(\Re \mathfrak{e}=$ Realteil $)$, die für genügend kleine Feldstärken (bis zu einigen Volt $\mathrm{cm}^{-1}$ ) durch die Näherung

$\gamma=12\left(\begin{array}{c}e \mathbf{a} F \\ \hbar \Gamma\end{array}\right)^{2} \Gamma=1,97 \cdot 10^{-3}\left(\frac{F}{\text { Volt cm }^{-1}}\right)^{2} \cdot \Gamma$

ersetzt werden kann. Auch hier ist natürlich der $\gamma$-Wert von $7 \mathrm{sec}^{-1}$ für die Emission zweier Quanten hinzu zu addieren.

Ein Blick auf die Abb. 2 und der Vergleich von (18) mit (20) zeigen, daß bei Feldstärken zwischen 0,03 und 10 Volt $\mathrm{cm}^{-1}$ infolge der Lamb-shift eine Verlängerung der mittleren Lebensdauer um mehr als zwei Zehnerpotenzen stattfindet. 


\title{
Ausbreitung elektrischer Wellen längs geschichteter und längs kontinuierlich veränderlicher Plasmen
}

\author{
Von W. O. Schumans
}

Aus dem Elektrophysikalischen Institut der Technischen Hochschule München

(Z. Naturforschg. 5 a, 612-617 [1950]; eingegangen am 21. Oktober 1950)

\begin{abstract}
Es wird die Wellenausbreitung längs eines geschichteten und längs eines kontinuierlich in seiner Dichte veränderlichen Plasmas beschrieben. Für ein Plasma aus 2 Schichten, von denen die innere dichter ist, ergeben sich in der Frequenz 2 Ausbreitungsbereiche, in denen jeweils die Phasengeschwindigkeit vom Werte $c$ bei der unteren Grenzfrequenz auf den Wert Null bei der oberen Frequenzgrenze sinkt. Ein Plasma, dessen Dichte linear nach innen beliebig hoch ansteigt, hat eine Grenzschichtwelle bei der Frequenz unendlich, wo sich die Welle sehr stark um die Stelle $\varepsilon=0$ herum konzentriert und die Phasengeschwindigkeit gegen Null geht. Für ein Plasma konstanter Dichte und der Eigenfrequenz $\omega_{0}$, mit einer in der Dichte linear nach außen abfallenden Übergangsschicht, sind Wellen nur für $\varepsilon_{\mathrm{P}}<-1$, d. h. $\omega^{2}<\omega_{0}^{2} / 2$ möglich. Je mehr sich die Welle um die Stelle $\varepsilon=0$ konzentriert, und je geringer ihre Phasengeschwindigkeit ist, um so näher liegt $\omega^{2}$ an $\omega_{0}^{2} / 2$. Die Dämpfung des Plasmas spielt eine sehr erhebliche Rolle.
\end{abstract}

\section{I. Über elektrische Wellen längs eines geschichteten Plasmas}

Tn einer Arbeit ${ }^{1}$ habe ich gezeigt, daß Wellen längs einer homogenen Plasmaschicht bzw. eines Plasmazylinders sich nur bei Frequenzen $\omega \equiv \omega_{0} / / 2$ $\left(\omega_{0}{ }^{2}=N e^{2} / m \varepsilon_{0}\right)$ ausbreiten, und daß die Phasen-

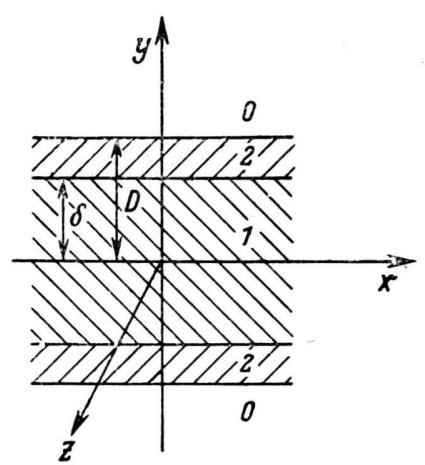

Abb. 1.

und Gruppengeschwindigkeit vom Werte $c$ bei $\omega=0$ bis auf den Wert Null bei der oberen Grenzfrequenz $\omega_{\mathrm{g}}=\omega_{0} / / 2$ abfällt.

Da begrenzte Plasmen niemals streng homogen sind, war noch zu diskutieren, ob dies auch bei Plasmen veränderlicher Dichte zutrifft.

Es soll deshalb zunächst im folgenden ein geschichtetes Plasma nach Abb. 1 untersucht werden.

Wir setzen für die Schichtung, zweidimensional, mit der Form $e^{i(\omega t-a x)}$ eine longitudinale $E$-Welle an:
1. in Luft

$$
\begin{gathered}
H_{z_{0}}=A_{0} e^{m_{0} y}, \quad E_{x_{0}}=i{ }_{\omega \varepsilon_{0}}^{m_{0}} A_{0} e^{-m_{0} y}, \\
E_{y_{0}}=\frac{\alpha}{\omega \varepsilon_{0}} A_{0} e^{-m_{0} y},
\end{gathered}
$$

$\varepsilon_{0}$ Dielektrizitätskonstante des Vakuums,

$$
\alpha^{2}-\omega^{2} / c^{2}=m_{0}{ }^{2} ;
$$

2. im äußeren Plasma 2

$$
\begin{aligned}
& H_{z_{3}}=A_{2} e^{m_{2} y}+B_{2} e^{m_{2} y}, \\
& E_{x_{2}}=j_{\omega \varepsilon_{0} \varepsilon_{2}}^{m_{2}}\left[A_{2} e^{m_{2} y}-B_{22} e^{-m_{2} y}\right], \\
& E_{y_{2}}=\underset{\omega \varepsilon_{0} \varepsilon_{2}}{a}\left[A_{2} e^{-m_{2} y}+B_{2} e^{-m_{2} y}\right],
\end{aligned}
$$

$\therefore=1-\omega^{2} 02 / \omega^{2}, \quad$ relative Dielektrizitätskonstante des Plasmas 2,

$$
\alpha^{2}-\frac{\omega^{2}}{c^{2}} \varepsilon_{2}=m_{2}{ }^{2},
$$

3. im inneren Plasma 1 ein in $E_{x}$ zu $y=0$ symmetrisches Feld

$$
\begin{aligned}
& H_{z_{1}}=-A_{1} \text { ङin } m_{1} y, \quad E_{x_{1}}=j \frac{m_{1}}{\omega \varepsilon_{0} \varepsilon_{1}} A_{1} \text { ( of } m_{1} y, \\
& E_{y_{1}}=-\underset{\omega \varepsilon_{0} \varepsilon_{1}}{\ll} A_{1} \operatorname{Sin} m_{1} y_{1} \text {, } \\
& \varepsilon_{1}=1-\frac{\omega^{2}{ }^{2}}{\omega^{2}}, \quad \alpha^{2}-\frac{\omega^{2}}{c^{2}} \varepsilon_{1}=m_{1}{ }^{2} .
\end{aligned}
$$

1 W. O. S chumann, S.-B. math.-naturwiss. Abt bayr. Akad. Wiss. 1948, 255. 
Dann ergeben die Stetigkeitsbedingungen an den Grenzen $y=\delta$ und $y=D$ die Gleichung

$-\mathfrak{T g} m_{1} \delta=\frac{m_{1}}{m_{2}} \frac{1}{\varepsilon_{1}} \frac{1+\epsilon_{2} \frac{m_{0}}{m_{2}} \mathfrak{T}_{\mathfrak{g}} m_{2}(D-\delta)}{1+\frac{1}{\varepsilon_{2}} \frac{m_{2}}{m_{0}} \mathfrak{T} \mathfrak{g} m_{2}(D-\delta)},$,

wozu noch für die $m$ die Gleichungen gelten

$$
m_{1}^{2}-m_{0}^{2}=\frac{\omega^{2}{ }_{01}}{c^{2}}, \quad m_{2}^{2}-m_{0}^{2}=\frac{\omega^{2}{ }_{02}}{c^{2}} .
$$

Es ist also $m_{1}>m_{2}>m_{0}$, wenn $\omega_{01}>\omega_{02}$ ist, d. h. in der Mitte das dichteste Plasma ist. Da $m_{0}$ positiv und reell sein muß, kommen imaginäre $m_{1}$ und $m_{2}$ nicht in Frage. Die "graphische " Lösung dieser Gleichung ist nur im dreidimensionalen $m_{0^{-}}, m_{1^{-}}$ und $m_{2}$-Raum möglich. Wir wollen dies hier nicht durchführen, sondern nur nach der Existenz der eingangs erwähnten beiden charakteristischen Grenzpunkte bei $\omega=0, v_{\mathrm{P}}=c$, und $v_{\mathrm{P}}=0$ bei einer oberen Grenzfrequenz fragen.

a) $v_{\mathrm{P}}=\omega / \alpha=0$ bei der oberen Grenzfrequenz, Bildung einer Grenzflächenwelle

In diesem Falle erwarten wir analog Arbeit ${ }^{1}, \mathrm{da} ß m_{1}$, $m_{2}$ und $m_{0}$ mit $\alpha$ sehr groß und von gleicher Größenordnung werden. Dann können wir die $\mathfrak{I} \mathfrak{g}$ gleich 1 setzen und aus der Stetigkeitsbedingung (I. 1) $m_{2} / m_{0}$ als Funktion von $m_{1} / m_{0}$ berechnen. Als Lösung ergibt sich

$$
\frac{m_{2}}{m_{0}}=-\frac{\varepsilon_{2}}{\varepsilon_{1}} \frac{m_{1}}{m_{0}} .
$$

Führt man nun nach (I. 2.)

$$
\begin{gathered}
m_{1}{ }^{2} / m_{0}{ }^{2}=1+\omega^{2}{ }_{01} / c^{2} m_{0}{ }^{2} \\
\text { und } m_{2}{ }^{2} / m_{0}{ }^{2}=1+\omega^{2}{ }_{02} / c^{2} m_{0}{ }^{2}
\end{gathered}
$$

in diese Gleichung ein, so entsteht

$$
\frac{1}{c^{2} m_{0}{ }^{2}}=\frac{\left(\varepsilon_{2} / \varepsilon_{1}\right)^{2}-1}{\omega^{2}{ }_{02}-\left(\varepsilon_{2} / \varepsilon_{1}\right)^{2} \omega^{2}{ }_{01}} .
$$

$m_{0}{ }^{2}$ wird also nur dann sehr groß, wenn

$$
\varepsilon_{2} / \varepsilon_{1}=-1
$$

ist, und gleichzeitig $\omega^{2}{ }_{02} \neq \omega_{01}^{2}$ ist.

Aus der Bedingung (I. 3.) folgt als obere Grenzfrequenz, bei der $\alpha \rightarrow \infty, v_{\mathrm{P}} \rightarrow 0$ geht

$$
\begin{gathered}
\omega_{\mathrm{g}}{ }^{2}=\frac{\omega^{2}{ }_{01}+\omega^{2}{ }_{02}}{2}, \quad \varepsilon_{1}=\frac{\omega^{2}{ }_{02}-\omega_{01}^{2}}{\omega^{2}}+\omega^{2}{ }_{01} \\
\varepsilon_{2}=\frac{\omega^{2}-\omega_{02}^{2}}{\omega^{2}+\omega^{2}} .
\end{gathered}
$$

Für $\omega_{01}>\omega_{02}$, d. h. innen dichteres Plasma, folgt im Plasmakern $\varepsilon_{1}<0$, und in der äußeren Plasmahülle $\varepsilon_{2}>0$. Die Felder im Kernplasma steigen sehr stark gegen den äußeren Rand an. In der äußeren Plasmahülle fallen sie nach außen sehr stark ab, da die Konstante $B$ sehr klein gegen $A$ wird. Die Schichtdicken spielen in diesem Fall keine Rolle, da die Welle sich so stark um die Grenze Kernplasma - Plasmahülle, $y=\delta$, konzentriert, daß die Dicke des Kerns und der Hülle keine Rolle spielen. Die Welle verläuft ganz innerhalb des Plasmas und in der äußeren Luft merkt man so gut wie nichts von ihr. Die Feldstärken $E_{y_{1}}$ und $E_{y_{2}}$ sind einander entgegengesetzt gerichtet, da $A_{1}$ das entgegengesetzte Vorzeichen hat wie $A_{2}$, $\varepsilon_{1}$ negativ und $\varepsilon_{2}$ positiv ist. Es treten also pulsierende Raumladungen an den Plasmagrenzen auf. Es ist wegen der Stetigkeit

$$
\frac{A_{2}}{A_{1}}=e^{m_{2} D} \frac{1+\frac{m_{0}}{m_{2}} \varepsilon_{2}}{1-\frac{m_{0}}{m_{2}} \varepsilon_{2}}
$$

und

$$
A_{1} \Xi \text { in } m_{1} \delta=-A_{2}\left[e^{-m_{2} \delta}+e^{-m_{2} D} \frac{1-\frac{m_{0}}{m_{2}} \varepsilon_{2}}{1+\frac{m_{0}}{m_{2}} \varepsilon_{2}}\right] .
$$

Damit ist eine Grenzflächenwelle innerhalb des Plasmas und eine obere Grenzfrequenz für $\omega_{01} \neq \omega_{02}$ nachgewiesen.

Es existiert aber noch eine tiefer liegende Grenzfrequenz mit $v_{\mathrm{P}}=0$, die aus (I. 1) folgt, wenn $\varepsilon_{2} m_{0} / m_{2} \rightarrow-1$ geht. Es wird dann der Bruch der Gl. (I. 1) unbestimmt, $\%$, wenn $m_{0}$ und $m_{2}$ mit gleicher Größe gegen $\infty$ gehen und gleichzeitig $\varepsilon_{2} \rightarrow-1$. Schreibt man die Gl. (I. 1) in der Form

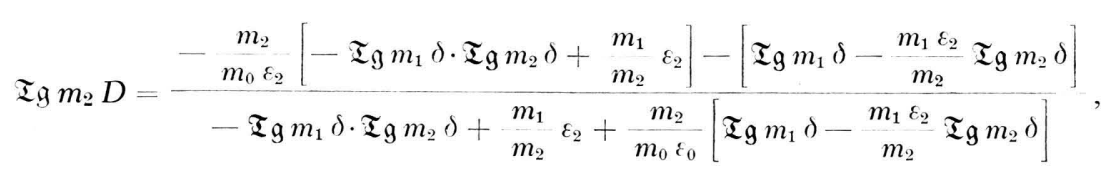


so sieht man, daß in diesem Fall Ig $m_{2} D \rightarrow 1$, d. h. $m_{2} \rightarrow \infty$ geht, mit $\varepsilon_{2} \rightarrow-1$, d. h. $\omega^{2} \rightarrow \omega_{02}^{2} / 2$ und daher auch $m_{0} \rightarrow \infty$. Es ist dies eine Grenzflächenwelle an der Grenze des äußeren Plasmas gegen die Luft. Wir haben also 2 obere Grenzfrequenzen mit $v_{\mathrm{P}}=0$ bei $\omega=\left(\omega^{2}{ }_{01}+\omega^{2}{ }_{02}\right) / 2$ und bei $\omega^{2}=\omega^{2}{ }_{02} / 2$.

b) Verhalten bei $\omega \rightarrow 0$

In diesem Fall erwarten wir nach Arbeit ${ }^{1}$, daß mit $\omega \rightarrow 0, m_{0}$ auch gegen Null geht, $v_{\mathrm{P}} \rightarrow \mathrm{c}$, wobei aber gleichzeitig $\varepsilon_{1}$ und $\varepsilon_{2}$ gegen $\infty$ gehen.

$$
\varepsilon_{1} \rightarrow-\omega^{2}{ }_{01} / \omega^{2}, \quad \varepsilon_{2} \rightarrow-\omega^{2}{ }_{02} / \omega^{2} .
$$

$m_{2}$ und $m_{1}$ bleiben endlich. Da auch $\alpha$ mit $\omega$ gegen Null geht, nähern sich mit verschwindender Frequenz

$$
m_{1}{ }^{2} \rightarrow \omega^{2}{ }_{01} / c^{2}, \quad m_{2}{ }^{2} \rightarrow \omega^{2}{ }_{02} / c^{2} .
$$

Mit diesen Voraussetzungen kann man aus der Stetigkeitsgleichung (I.1) $m_{0} \varepsilon_{1}$ berechnen und erhält

$$
\begin{array}{r}
-m_{0} \varepsilon_{1}=m_{0} \frac{\omega^{2}{ }_{01}}{\omega^{2}} \\
=\frac{\omega_{01}}{c} \frac{\frac{\omega_{01}}{\omega_{02}} \mathfrak{I g} m_{1} \delta \mathfrak{I g}_{\mathfrak{g}} m_{2}(D-\delta)+1}{\mathfrak{T g} m_{1} \delta+\frac{\omega_{01}}{\omega_{02}} \mathfrak{T g}_{\mathrm{g}} m_{2}(D-\delta)} .
\end{array}
$$

Hieraus kann $m_{0}$ berechnet werden und man erkennt, daß es proportional mit $\omega^{2}$ gegen Null geht. Für $\omega_{01}=\omega_{02}$ geht die Gleichung über in

$$
m_{0}=\frac{\omega^{2}}{\omega_{0} c} \frac{1}{\operatorname{Tg} m_{1} D}
$$

wie in Arbeit ${ }^{1}$ für homogenes Plasma abgeleitet.

Mit abnehmender Frequenz nähert sich die Phasengeschwindigkeit der Lichtgeschwindigkeit. Das Feld erstreckt sich sehr weit in den Luftraum und ist innerhalb der Plasmen nur wenig veränderlich.

c) Lichtgeschwindigkeit bei endlicher Frequenz

Nicht nur bei $\omega=0$ wird $v_{\mathrm{P}}=c$, sondern auch für endliches $\omega$ mit $m_{0}=0, a=\omega / c, m_{1}{ }^{2}=\omega^{2}{ }_{01} / c^{2}$, $m_{2}{ }^{2}=\omega^{2}{ }_{02} / c^{2}$ folgt aus (I.1) für endliche $\varepsilon_{1}$ und $\varepsilon_{2}$

$$
-\mathfrak{T} \mathfrak{g} m_{1} \delta \mathfrak{T g} m_{2}(D-\delta)=\frac{m_{1}}{m_{2}} \frac{\varepsilon_{2}}{\varepsilon_{1}}
$$

und aus dieser Gleichung ergibt sich $\varepsilon_{2} / \varepsilon_{1}$ und daraus die Frequenz.

Da $m_{1} \delta$ und $m_{2}(D-\delta)$ in diesem Fall meist klein gegen 1 sind, kann man $\mathfrak{I g}$ durch die Argu- mente ersetzen und erhält dann

und daraus

$$
-\delta(D-\delta) \frac{\omega^{2}{ }^{2}}{c^{2}}=\frac{\varepsilon_{2}}{\varepsilon_{1}}
$$

$$
\omega^{2}=\omega^{2}{ }_{02} \frac{1+\delta(D-\delta) \omega^{2} / c^{2}}{1+\delta(D-\delta) \omega_{02}^{2} / c^{2}} .
$$

Es ist also bei $\omega_{01}>\omega_{02}, \omega^{2}>\omega^{2}{ }_{02}$ aber $\omega^{2}<\omega^{2}{ }_{01}$, i. h. $\varepsilon_{2}>0$ und $\varepsilon_{1}<0$.

Wir haben also 2 Frequenzbänder zu erwarten. Das erste von $\omega^{2}=0$ bis $\omega^{2}{ }_{02} / 2$ und das zweite von

$$
\omega^{2}=\omega^{2}{ }_{02} \frac{1+\delta(D-\delta) \omega_{01}^{2} / c^{2}}{1+\delta(D-\delta) \omega^{2}{ }_{02} / c^{2}}
$$

bis $\omega^{2}=\left(\omega^{2}{ }_{01}+\omega^{2}{ }_{02}\right) / 2$. In beiden geht die Phasengeschwindigkeit von $v_{\mathrm{P}}=c$ an der unteren Grenze bis auf Null bei der oberen Grenze.

\section{Plasma mit kontinuierlicher Dichteverteilung}

Wenn die Dichteverteilung eines Plasmas entsprechend Abb. 1 kontinuierlich in $y$-Richtung sich ändert, so ergibt sich für $(\operatorname{Tr} H)$ longitudinale $E$-Wellen, die in $x$-Richtung laufen, mit dem Ansatz $e^{j(\omega t-a x)}$ die Differẹntialgleichung für $H_{z}$

$$
\begin{gathered}
\frac{\partial^{2} H_{z}}{\partial y^{2}}-\frac{1}{\varepsilon} \frac{\partial \varepsilon}{\partial y} \frac{\partial H_{z}}{\partial y}+\left(\frac{\omega^{2}}{c^{2}} \varepsilon-c^{2}\right) H_{z}=0, \\
\varepsilon=1-\omega_{0}{ }^{2} / \omega^{2}, \quad \omega_{0}{ }^{2}=\frac{N e^{2}}{m \varepsilon_{0}}, \quad \varepsilon=f(y)
\end{gathered}
$$

und

$$
E_{x}=-\frac{j}{\omega \varepsilon_{0} \varepsilon} \frac{\partial H_{z}}{\partial y}, \quad E_{y}=\frac{a}{\omega \varepsilon_{0} \varepsilon} H_{z}
$$

Die allgemeine Lösung dieser Gleichung wird an anderer Stelle diskutiert werden. Eine brauchbare Näherung für viele Zwecke ist die Annahme $a^{2} \gg \omega^{2} \varepsilon / c^{2}$ oder $v \mathrm{P}^{2} / c^{2} \ll 1 / \varepsilon$, die besonders in der Gegend kleiner $v_{\mathrm{P}}$ und kleiner $\varepsilon$ gut zutrifft, da $v_{\mathrm{P}}$ normalerweise $<c$ ist.

Nimmt man einen linearen Verlauf der Dielektrizitätskonstante an, z. B. $\varepsilon=y a$, so wird aus (II.1)

$$
\frac{\partial^{2} H_{z}=1}{\partial y^{2}-y} \frac{\partial H_{z}}{\partial y}+\left(\frac{\omega^{2}}{c^{2}} a y-a^{2}\right) H_{z}=0 .
$$

In diesem Fall ist für $y=1 / a, \omega_{0}^{2}=0, \varepsilon=1$ und für $y=0, \varepsilon=0$, ist $\omega_{0}{ }^{2}$ auf $\omega^{2}$ angestiegen. Für negatives $y$ ist $\omega^{2}<\omega_{0}{ }^{2}$. Für linearen Anstieg der Plasmadichte $-d \omega_{0}^{2} / d y=k$ ist

$$
a=k / \omega^{2},
$$


d. h. für tiefe Frequenzen liegt $\varepsilon=0$ nahe am Plasmarand, dagegen für hohe Frequenzen tief innerhalb des Plasmas. Setzt man wieder $a y \omega^{2} / c^{2} \ll \alpha^{2}$, d. h. $a y \ll c^{2} / v_{\mathrm{P}}{ }^{2}$ voraus, so vereinfacht sich die Gleichung zu

$$
\frac{\partial^{2} H_{z}}{\partial y^{2}}-\frac{1}{y} \frac{\partial H_{z}}{\partial y}-a^{2} H_{z}=0
$$

und hat die Lösung $H_{z}=y Z_{1}(j a y)$, wo $Z$ eine Besselsche Funktion ist. Damit wird

$$
E_{x}=\frac{\varepsilon}{\omega \varepsilon_{0} a} Z_{0}(j a y) \text { und } E_{y}=\frac{\alpha}{\omega \varepsilon_{0} a} Z_{1}(j a y) .
$$

Für die angrenzende Luft setzen wir wieder

$$
H_{z_{0}}=A_{0} e^{-m_{0} y}, \quad E_{x_{0}}=j \frac{m_{0}}{\omega \varepsilon_{0}} A_{0} e^{--m_{0} y} .
$$

Aus der Stetigkeit von $H_{z}$ und $E_{x}$ für $y=1 / a$ folgt dann

$$
j m_{0}=i \sqrt{\alpha^{2}-\frac{\omega^{2}}{c^{2}}=\alpha \frac{Z_{0}(j a / a)}{Z_{1}(j \alpha / a)} .} .
$$

Da die Felder in die Tiefe des Plasmas hinein für große negative $y$ exponentiell verschwinden müssen, kommt für $y<0$ als Lösung nur die Hankelsche Funktion 2. Art in Frage. Andererseits läßt sich Gl. (II. 6) nur für Hankelsche Funktionen 1. Art erfüllen, die also für $y>0 \mathrm{zu}$ verwenden sind. Dies stört die Stetigkeit von $E_{x}$ und $H_{z}$ bei $y=0$ nicht, da beide Hankelsche Funktionen in gleicher Weise dort $\infty$ werden. $H_{0}{ }^{1}(x) \rightarrow H_{0}{ }^{2}(-x)$ für $x \rightarrow 0$, während $E_{y}$ rechts und links von der Stelle $x=0$ entgegengesetztes Vorzeichen hat, $H_{1}{ }^{1}(x) \rightarrow-H_{1}{ }^{2}(-x)$ für $x \rightarrow 0$.

Man setzt also für $0<y<1 / a$

$$
\begin{gathered}
H_{z}=y A H_{1}{ }^{1}(j \alpha y), \quad E_{x}=\frac{\alpha}{\omega \varepsilon_{0} a} A H_{0}{ }^{1}(j \alpha y), \\
E_{y}=\frac{a}{\omega \varepsilon_{0} a} A H_{1}{ }^{1}(j \alpha y)
\end{gathered}
$$

und für $y<0$

$$
\begin{gathered}
H_{z}=y A H_{1}{ }^{2}(j \alpha y), \quad E_{x}=\frac{\alpha}{\omega \varepsilon_{0} a}{ }^{2} A H_{0}{ }^{2}(j \alpha y), \\
E_{y}=\frac{\alpha}{\omega \varepsilon_{0} a} A H_{1}{ }^{2}(j \alpha y) .
\end{gathered}
$$

Aus diesen Ansätzen folgt, daß für $y \rightarrow 0, \varepsilon \rightarrow 0$, $H_{z}$ dem Wert $A / j a$ zustrebt, während die elektrischen Felder theoretisch $\infty$ groß werden, d. h. bei der stets vorhandenen Dämpfung einem Maximalwert zustreben. Da aber $E_{x}$ nur logarithmisch $\infty$ wird, $E_{y}$ dagegen wie $1 / y$, ist nahe $\varepsilon=0, E_{y}$ sehr groß gegen
$E_{x}$, so daß die Kraftlinien die Stelle $y=0$ praktisch senkrecht, parallel zu $y$ durchsetzen. Außerdem hat $E_{y}$ oberhalb und unterhalb von $y=0$ entgegengesetztes Vorzeichen und nimmt nach beiden Seiten sehr viel rascher ab als $E_{x}$. Es führt dies zu einem Verlauf der Kraftlinien, wie in Abb. 2 skizziert, und wie er nach Arbeit ${ }^{1}$ auch zu erwarten ist.

Bei $y=0$ bilden sich starke pulsierende Raumladungen, die durch die gegenphasigen Verschiebungen der Elektronen' beiderseits von $y=0$ erzeugt werden.

Die Grenzbedingung (II. 6) heißt dann

$$
j_{0} m=i \sqrt{\alpha^{2}-\frac{\omega^{2}}{c^{2}}}=\alpha \frac{H_{0}{ }^{1}(j \alpha / a)}{H_{1}{ }^{1}(j \alpha / a)},
$$

aus der zu jedem $\omega$ das zugehörige $\alpha$ bestimmt werden kann, wobei $\alpha^{2} \gg \omega^{2} / c^{2}$ vorausgesetzt ist. Fragt

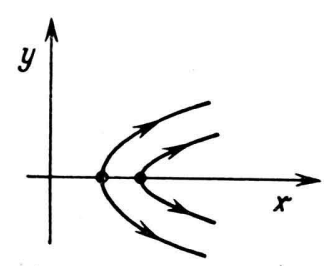

Abb. 2.

man speziell nach großen Werten von $\alpha, \alpha \rightarrow \infty$, $v_{\mathrm{P}} \rightarrow 0$, so ergibt sich für große $z$ mit

$$
\begin{gathered}
H_{0}{ }^{1}(j z)=-i \frac{e^{-z}}{\sqrt{\frac{\pi}{2} z}}\left(1-\frac{1}{8 z}\right), \\
H_{1}{ }^{1}(j z)=-\frac{e^{-z}}{\sqrt{\frac{\pi}{2} z}}\left(1+\frac{3}{8 z}\right), \\
\frac{\omega^{2}}{c^{2}}=\frac{5}{8} a a+\frac{a^{2}}{4} .
\end{gathered}
$$

Mit wachsendem $\alpha$ muß auch $\omega$ wachsen. Mit $a=k / \omega^{2}$ (II.5) ergibt sich

oder

$$
\frac{\omega^{2}}{c^{2}}=\frac{5}{8} \frac{k}{\omega^{2}} \alpha+\frac{k^{2}}{\omega^{4}}
$$

$$
\frac{\omega}{c} \approx \sqrt[4]{\frac{5}{8} \frac{k \alpha}{c^{2}}}
$$

und die Phasengeschwindigkeit wird

$$
v_{\mathrm{P}}=\frac{\omega}{\alpha} \approx c \sqrt[4]{\frac{5}{8} \frac{k}{\alpha^{3} c^{2}}} .
$$


Es gibt auch in diesem Fall mit $a \rightarrow \infty$ eine ausgeprägte Grenzflächenwelle mit sehr stark abfallenden Feldern oberhalb und unterhalb von $y=0$, $\varepsilon=0$, und von sehr geringer Phasengeschwindigkeit. Nur tritt sie hier erst bei sehr hohen Frequenzen auf. Je steiler die Plasmadichte ansteigt, desto größer wird $v_{\mathrm{P}}$ bei gegebenem $\alpha$.

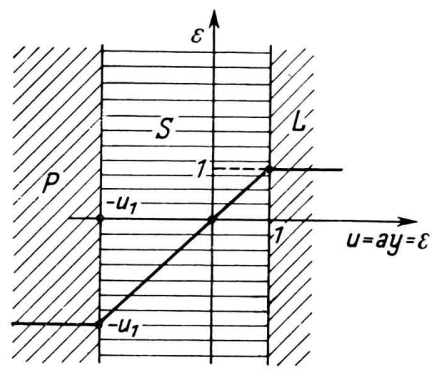

Abb. 3. P Plasma konstanter Dichte, S Übergangsschicht, L Luft.

Für den Fall, daß die mit abnehmendem $y$ anwachsende Plasmadichte schließlich wieder konstant wird, genügt die angegebene Näherung nicht zur Befriedigung der Grenzbedingungen. Dagegen können wir in diesem Fall eine andere Näherung verwenden, diè einen gewissen Einblick in die Verhältnisse gibt. Führt man in die Differentialgleichung 4 als neue unabhängige Variable $u=a y=\varepsilon$ ein, so entsteht

$$
\frac{\partial^{2} H_{z}}{\partial u^{2}}-\frac{1}{u} \frac{\partial H_{z}}{\partial u}+\frac{1}{a^{2}}\left(\frac{\omega^{2}}{c^{2}} u-\alpha^{2}\right) H_{z}=0 .
$$

Ist nun $a^{2}$ genügend viel größer als $u \omega^{2} / c^{2}-a^{2}$, so kann das letzte Glied dieser Gleichung vernachlässigt werden, und man erhält

$$
\begin{gathered}
H_{z}=C_{1} \frac{u^{2}}{2}+C_{2}, \quad E_{x}=-j \frac{C_{1}}{\omega \varepsilon_{0}}, \\
E_{y}=\frac{\alpha}{\omega \varepsilon_{0}}\left(C_{1} \frac{u}{2}+\frac{C_{2}}{u}\right) .
\end{gathered}
$$

Es ist jetzt $E_{x}$ in der Übergangsschicht konstant, $H_{z}$ wächst symmetrisch zu $u=0$ und $E_{y}$ ist antisymmetrisch zu $u=0$ und geht für $u \rightarrow 0$ gegen $\infty$.

Nimmt man nun einen Plasmaverlauf nach Abb. 3 an, d. h. von $u=1$ bis $u=-u_{1}$ einen linearen Verlauf der Dielektrizitätskonstante von 1 über 0 bis $-u_{1}$, und daran anschließend ein Plasma konstanter Dichte mit der Dielektrizitätskonstante $\left(-u_{1}\right)$, so gilt innerhalb der Luft für $u>1$

$$
H_{0}=A_{0} e^{-m_{0} y}, \quad E_{x_{1}}=j \frac{m_{0}}{\omega \varepsilon_{0}} A_{0} e^{-m_{0} y}
$$

und im homogenen Plasma für $u<-u_{1}$, mit $u_{1}{ }^{2}=\varepsilon^{2} \mathrm{P}$,

mit

$$
H_{z_{1}}=A_{1} e^{m_{1} y}, \quad E_{x_{1}}=-i \frac{m_{1}}{\omega \varepsilon_{0} \varepsilon_{\mathrm{P}}} A_{1} e^{m_{1} y}
$$

$\varepsilon_{\mathrm{P}}=1-\frac{\omega_{0}{ }^{2}}{\omega^{2}}, \quad m_{0}{ }^{2}=\alpha^{2}-\frac{\omega^{2}}{c^{2}}, \quad m_{1}{ }^{2}=\alpha^{2}-\frac{\omega^{2}}{c^{2}}{ }^{2} \mathrm{P}$,

$$
m_{1}{ }^{2}-m_{0}{ }^{2}=\frac{\omega_{0}{ }^{2}}{c^{2}} .
$$

Dann folgt aus den Stetigkeitsbedingungen bei $u=1$ und $u=-u_{1}$

$$
m_{0}=-\frac{C_{1}}{\frac{C_{1}}{2}+C_{2}} \text { und } \frac{m_{1}}{\varepsilon_{\mathrm{P}}}=\frac{C_{1}}{C_{1} \frac{u_{1}^{a^{2}}+C_{2}}{2}}
$$

oder

$$
\frac{1}{m_{0}}+\frac{{ }^{\varepsilon} \mathrm{P}}{m_{1}}=\frac{{ }^{\varepsilon_{\mathrm{P}}}{ }^{2}-1}{2} .
$$

Da positives $\varepsilon_{\mathrm{P}}<1$ ist, ist diese Gleichung für positive $m_{0}$ und $m_{1}$ nur für negatives $\varepsilon_{\mathrm{P}}$ lösbar. In dem homogenen Plasma ist $\omega^{2}<\omega_{0}{ }^{2}$. Mit $-\varepsilon \mathrm{P}=\varepsilon_{\mathrm{P}}{ }^{\prime}$ ist dann

$$
m_{0}=\frac{1}{\left(\varepsilon_{\mathrm{P}}^{\prime}-1\right) 2+\varepsilon_{\mathrm{P}}{ }^{\prime} / m_{1}} .
$$

Diese Beziehung ist in Abb. 4 als Kurve 1 graphisch dargestellt.

Da außerdem die Beziehung $m_{1}{ }^{2}-m_{0}{ }^{2}=\omega_{0}{ }^{2} / c^{2}$ gilt (Abb.4, Kurve 2), gibt der Punkt $\mathrm{P}$ die zusammengehörigen Werte von $m_{0}$ und $m_{1}$ an.

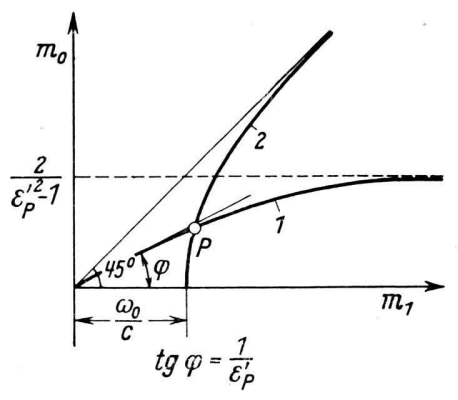

Abb. 4 .

Man sieht, daß nur $\varepsilon \mathrm{p}^{\prime}>1$ mögliche Werte ergibt, d. h. $\omega_{0}{ }^{2} / \omega^{2}>2$, und daß große $m_{0}$ und $m_{1}$ nur für $c \mathrm{p}^{\prime}$ nahe an 1 auftreten. Allerdings gilt die Lösung nur unter der Bedingung

die sich auch als

$$
a^{2} \gg\left|\frac{\omega^{2}}{c^{2}} u-\alpha^{2}\right|,
$$

$$
a^{2} \gg\left|\frac{\omega_{0}{ }^{2}}{c^{2}}+m_{0}{ }^{2}\right|
$$


schreiben läßt. Für $m_{0}$ sehr groß, muß auch $a$ sehr groß werden, d. h. für die Grenzflächenwelle $\alpha \rightarrow \infty$, $m_{0} \rightarrow \infty$ geht auch $a \rightarrow \infty$, d. h. wir kommen in diesem Fall wieder auf die homogene und unstetig in Luft übergehende Plasmaschicht zurück. Ob eine Grenzflächenwelle bei endlicher Frequenz auch bei einer Übergangsschicht ( $a$ endlich) auftritt, kann demnach diese Näherungslösung nicht entscheiden. Da $\alpha^{2}=m_{0}{ }^{2}+\omega^{2} / c^{2}$ ist, treten Phasengeschwindigkeiten

$$
v_{\mathrm{P}}^{2}=c^{2} \frac{1}{1+m_{0}^{2} c^{2} / \omega^{2}}
$$

kleiner als die Lichtgeschwindigkeit auf, die um so kleiner sind, je größer $m_{0}$ ist und um so rascher auf beiden Seiten der Übergangsschicht abfallen, je größer $m_{0}$ und $m_{1}$ sind.

\title{
Über die Beugung von Rohrwellen an ebenen Blenden
}

\author{
Von RolF MÜlleR \\ Aus dem Institut für theoretische Physik der Technischen Hochschule München \\ (Z. Naturforschg. 5 a, 617-621 [1950]; eingegangen am 12. Oktober 1950)
}

\begin{abstract}
Im ersten einleitenden Abschnitt werden bekannte Beziehungen, die für das Folgende notwendig sind, zusammengestellt. Im zweiten Abschnitt wird das Beugungsproblem für Rohrwellen an ebenen Blenden allgemein formuliert. Im dritten Abschnitt wird für den speziellen Fall der konzentrischen Lochblende im kreiszylindrischen Rohr gezeigt, daß die Beugung einer einfallenden zylindersymmetrischen $E$ - bzw. $H$-Welle durch $E$ - bzw. $H$-Wellen allein beschrieben wird, also an der Blende keine Umwandlungen von $E$ - in $H$-Wellen auftreten, während bei einer einfallenden $E$ - bzw. $H$-Welle die vom Azimut $\varphi$ abhängt, zur Beschreibung der Beugung beide Wellentypen notwendig sind, also Umwandlungen auftreten.
\end{abstract}

I.

$\mathrm{B}_{\mathrm{r}}^{\mathrm{e}}$ ekanntlich läßt sich jeder mit der Frequenz $\omega$ periodische, elektromagnetische Feldzustand in einem ideal leitenden Hohlrohr durch 2 skalare Felder $e(\xi, \eta, z)$ und $h(\xi, \eta, z)$ in folgender Weise ausdrücken ${ }^{1}$ :

$$
\begin{aligned}
& \sqrt{\Delta} \xi=\left(\operatorname{grad}^{\prime} \frac{\partial}{\partial z} e+k \operatorname{grad}^{\prime} h \times j\right. \\
& \left.-j \operatorname{div} \operatorname{grad}^{\prime} e\right) e^{i \omega t}, \\
& i\rceil / I \mathfrak{Y}=\left(\operatorname{grad}^{\prime} \frac{\partial}{\partial z} h+k \operatorname{grad}^{\prime} e \times 3\right. \\
& \left.-\jmath \operatorname{div} \operatorname{grad}^{\prime} h\right) e^{i \omega t} \text {. }
\end{aligned}
$$

Die Striche an den Differentialoperatoren deuten an, daß in einer Querschnittsfläche $z=$ const., also nur nach den Koordinaten $\xi, \eta$ der Querschnittsfläche zu differenzieren ist. 3 ist der Einheitsvektor in Richtung der Rohrachse, $I$ und $\Delta$ sind die Materialkonstanten des Dielektrikums im Rohr und $k=\omega / \Pi / \Delta$ die Wellenzahl. Die Potentiale $e$ und $h$ müssen im Inneren des Hohlrohrs den Wellengleichungen:

$$
\triangle e+k^{2} e=0, \quad \triangle h+k^{2} h=0
$$

1 Siehe etwa E. Ru ch, Ann. Physik 7, 248 [1950]. und auf dem Rohrmantel den Randbedingungen

$$
e=0, \quad \frac{\partial h}{\partial n}=0
$$

genügen. Man verifiziert leicht, daß die durch die Gln. (1) bis (3) definierten Felder $\tilde{\mathfrak{S}}$ und $\mathfrak{\mathfrak { C }}$ im Inneren des Rohres den Maxwellschen Gleichungen

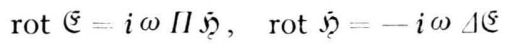

genügen und auf dem Rohrmantel die Tangentialkomponente des elektrischen Feldes $\tilde{E}_{t g}$ verschwindet. Man bezeichnet Schwingungszustände mit verschwindendem Potential $h$ als Schwingungen vom elektrischen Typus und solche mit verschwindendem Potential $e$ als Schwingungen vom magnetischen Typus. Die allgemeinsten Lösungen der Gln. (2) lauten

$$
\begin{aligned}
& e=\sum_{v^{\prime}}\left(a_{v^{\prime}}^{(e)} e^{i \gamma_{v}^{(e)} z}+\mathrm{b}_{v^{\prime}}^{(e)} e^{\left.-i \gamma_{v}^{(e)} z\right)} \varphi_{v^{\prime}},\right. \\
& h=\sum_{v^{\prime}}\left(a_{v}^{(h)} e^{i \gamma_{v}^{(h)} z}+b_{v}^{(h)} e^{-i \gamma_{v}^{(h)} z}\right) \psi_{v} .
\end{aligned}
$$

Dabei bedeuten die $a_{v}^{(e)}, b_{v}^{(e)}, a_{v}^{(h)}, b_{v}^{(h)}$ willkürliche komplexe Konstanten, mit denen man die Lösung vorgegebenen Randbedingungen anpassen kann. Die $\varphi_{1}, \psi_{1}$, die nur von den Koordinaten $\xi, \eta$ der Quer- 